\title{
Effect of S-Adenosyl-L-Methionine on Liver Biochemistry and Quality of Life in Patients with Primary Biliary Cholangitis Treated with Ursodeoxycholic Acid. A Prospective, Open Label Pilot Study
}

\author{
Ewa Wunsch ${ }^{1}$, Joanna Raszeja-Wyszomirska ${ }^{2}$, Olivier Barbier ${ }^{3}$, Malgorzata Milkiewicz ${ }^{4}$, Marcin Krawczyk ${ }^{5,6}$, \\ Piotr Milkiewicz ${ }^{1,2}$
}

1) Translational Medicine Group, Pomeranian Medical University, Szczecin, Poland 2) Liver and Internal Medicine Unit, Medical University of Warsaw, Warsaw, Poland 3) Laboratory of Molecular Pharmacology, CHU Quebec and Faculty of Pharmacy, Laval University, Quebec, Canada

4) Department of Medical Biology, Pomeranian Medical University, Szczecin, Poland 5) Department of Medicine II, Saarland University Medical Center, Saarland University, Homburg, Germany

6) Laboratory of Metabolic Liver Diseases, Department of General, Transplant and Liver Surgery, Medical University of Warsaw, Warsaw, Poland

\footnotetext{
Address for correspondence: Piotr Milkiewicz MD, MRCP(UK)

Liver and Internal Medicine Unit, Medical University of Warsaw, 02-097 Warsaw, Poland p.milkiewicz@wp.pl
}

Received: 20.04.2018 Accepted: 14.06 .2018

\section{ABSTRACT}

Background \& Aims: Chronic liver disease induces an acquired deficiency of S-adenosyl-L-methionine (SAMe) leading to impairment of detoxifying processes in the liver. Ursodeoxycholic acid (UDCA) represents the standard treatment in primary biliary cholangitis (PBC). As both compounds exert their hepatoprotective effects by different mechanisms, it is conceivable that when used together their effect might be additive. The aim of this study was to analyse the effect of SAMe supplementation on liver biochemistry and health-related quality of life (HRQoL) in patients with PBC, treated with UDCA.

Methods. In this prospective pilot, proof of the principle, non-randomized and open label study we enrolled 24 patients with PBC treated with UDCA for at least 6 months. They had received both UDCA in a standard dose of $13-15 \mathrm{mg} / \mathrm{kg}$ b.w. and SAMe in the dose of $1200 \mathrm{mg}$ daily over a period of 6 months. A group of 24 patients with $\mathrm{PBC}$ treated with UDCA served as control for liver biochemistry (Study registered on the platform ClinicalTrials.gov under ID: NCT02557360).

Results. We observed a significant decrease of ALP, GGT and total cholesterol in non-cirrhotic patients treated with SAMe. There was also a significant improvement of fatigue and pruritus in PBC-40 questionnaire and amelioration of anxiety in STAI 2 questionnaire in the SAMe group. Treatment with SAMe neither increased sulfation capacity of the liver nor had an effect on fibroblast growth factor-19 serum levels.

Conclusions. Our pilot study demonstrates a positive effect of adding SAMe to UDCA in non-cirrhotic patients with PBC.

Key words: primary biliary cholangitis - S-adenosyl-L-methionine - ursodeoxycholic acid - health-related quality of life - liver biochemistry.

Abbreviations: ALP: alkaline phosphatase; ALT: alanine aminotransferase; AST: aspartate aminotransferase; FGF-19: fibroblast growth factor-19; GGT: gammaglutamyl-transpeptidase; HADS: Hospital Anxiety and Depression Scale; HRQoL: health-related quality of life; INR: international normalized ratio; LCA: lithocholic acid; LCA-S sulfated LCA; LC-MS/MS: liquid chromatography/tandem mass spectrometry; MFIS: Modified Fatigue Impact Scale; PBC: primary biliary cholangitis; SAMe: S-adenosyl-L-methionine; SSRI: selective serotonin reuptake inhibitor; STAI: State-Trait Anxiety Inventory; UDCA: urosodeoxycholic acid.

\section{INTRODUCTION}

Primary biliary cholangitis (PBC) is a chronic cholestatic liver condition affecting predominantly middle-aged women. It may exert a significant negative effect on the patient's health-related quality of life (HRQoL) manifesting itself with intense pruritus and chronic fatigue in a substantial proportion of patients [1]. Ursodeoxycholic acid (UDCA) remains a standard therapy for PBC. However, a significant proportion of patients do not respond to this treatment [2]. S-adenosyl-L-methionine (SAMe) is involved in numerous biochemical reactions, playing a role of a major methyl and sulfate group donor and a precursor of cysteine and glutathione [3]. Chronic liver disease induces an acquired deficiency of methionine adenosyltransferase, which catalyzes SAMe formation from methionine leading to a depletion of the amount of SAMe and potential impairment of detoxifying processes in the liver [3]. SAMe as a single agent has been used in the past for the treatment of chronic liver diseases with equivocal results $[4,5]$. Our previous experimental works demonstrated that simultaneous treatment with UDCA 
and SAMe may exert beneficial, additive effect ameliorating cholestasis [6]. We postulated that hepatoprotective effects of SAMe may result from its role as a precursor of sulfate groups for detoxification processes in the liver $[7,8]$.

In this prospective, pilot, proof of the principle and open label study we analyzed the effect of the simultaneous treatment of UDCA and SAMe on liver biochemistry and HRQoL in patients with $\mathrm{PBC}$.

\section{MATERIAL AND METHODS}

We prospectively enrolled 24 consecutive patients with PBC, all female, treated with UDCA in a standard dose over a period of at least 6 months. All patients were recruited at two Polish Universities (Medical University of Warsaw, Warsaw and Pomeranian Medical University, Szczecin) between October 2015 and October 2016. The diagnosis of PBC was established according to the European Association for the Study of Liver criteria [2]. The severity of liver fibrosis was estimated by Shear Wave Elastography (SuperSonic Imagine; Aixplorer, Aix-en-Provence, France) performed by a single, experienced professional. A cut-off value for the diagnosis of liver cirrhosis was set at $13.0 \mathrm{kPa}$ according to a recent metaanalysis [9]. Patients with concomitant liver diseases, taking immunosuppressive, antipruritic, antihyperlipidemic and antidepressive drugs three months prior to baseline recording were considered not suitable for the study. Beside UDCA in the dose of $13-15 \mathrm{mg} / \mathrm{kg}$ b.w., all patients received vitamin $\mathrm{D}$ and calcium supplementation.

Participants received SAMe $1200 \mathrm{mg}$ /daily in two divided doses (800 $\mathrm{mg}$ in the morning and $400 \mathrm{mg}$ midday) over the period of 6 months. In both study centres they were monitored by experienced hepatologists in out-patient clinics at baseline, interim visits at weeks: $2,4,12$, end of treatment at 24 weeks and follow-up visit after 4-6 weeks wash-out period. Treatment and intervention adherence, adverse events, serum biochemistry and HRQoL were assessed at each visit.

Laboratory assessments encompassed routine serum biochemistry including alanine (ALT) and aspartate aminotransferases (AST), gammaglutamyl-transpeptidase (GGT), alkaline phosphatase (ALP), total bilirubin, albumin, international normalized ratio (INR) and total cholesterol.

In order to investigate the effect of SAMe on liver sulfation capacities, serum levels of lithocholic acid (LCA) and sulfated LCA (LCA-S) were measured using validated liquid chromatography/tandem mass spectrometry (LC-MS/ MS) method $[10,11]$. For each patient, LCA/LCA-S ratio was subsequently calculated.

As we previously showed, fibroblast growth factor-19 (FGF19), an important regulatory factor of bile acids synthesis, may serve as a valuable indicator of the cholestatic liver injury [12]. Therefore, in order to evaluate the potential hepatoprotective role of SAMe in cholestasis, we measured FGF-19 serum levels in the study group at the baseline and end-point as we described before [12].

HRQoL was assessed with a disease-specific questionnaire (PBC-40) [13, 14]. Chronic fatigue was measured by Modified Fatigue Impact Scale (MFIS) [15]. Emotional distress was assessed with Hospital Anxiety and Depression Scale (HADS) [16] and State-Trait Anxiety Inventory (STAI).

Four patients dropped-out from the study (one patient 2 weeks after inclusion, three others 4 weeks after inclusion) due to non-specific symptoms including abdominal pain or cramps. As the study protocol did not permit antipruritic therapies two other patients who complained of significant itching at baseline and who did not notice any improvement during the treatment were also removed from the study (at 4 weeks) in order to implement antipruritic therapy. Thus, 18 patients (10 with cirrhosis) aged 38-65 years (mean 54.2 \pm 8.2 ) were finally included.

Twenty four age-matched, consecutive female patients with PBC treated with UDCA for a period of at least 6 months served as controls for a comparison of liver biochemistry.

\section{Ethics}

Written informed consent was obtained from each patient included in the study. The study was performed following the principles of good clinical practice and in accordance with the ethical guidelines of the 1975 Declaration of Helsinki (6th revision, 2008). The study protocol was approved by the Ethics Committee of Pomeranian Medical University and Medical University of Warsaw. The study was registered on the platform ClinicalTrials.gov under ID: NCT02557360.

\section{Statistical analysis}

Descriptive statistical methods were used to analyze all variables. Continuous variables were evaluated using the number of observations, arithmetic mean and standard deviation (SD). Categorical data are described using absolute frequencies. To evaluate the changes in serum liver function tests we calculated delta values from baseline to the end of treatment visit for each patient. The Shapiro-Wilk normality test was used to examine the distribution of quantitative variables. The Wilcoxon matched-pairs signed rank test and the paired $t$-test were applied as appropriate to calculate the differences between variables at single time points. Pearson correlation coefficients and chi-square test were used to check the variables influencing SAMe biochemical response. To compare the differences between study and control groups unpaired $t$-test or Mann-Whitney test were applied. Calculations were performed using GraphPad Prism for Windows (Version 7.0). A p value less than 0.05 was considered statistically significant.

\section{RESULTS}

The baseline demographic and liver biochemistry data of study and control group are presented in Table I. At the enrolment there were no significant differences between the study and the control group.

\section{Effect of SAMe on liver biochemistry}

When baseline and 6 months results were compared, a significant improvement in ALP, GGT and cholesterol levels was seen in non-cirrhotic patients treated with SAMe. These data are shown in Fig. 1. In patients with cirrhosis, a significant 
Table I. Patients' baseline characteristics.

\begin{tabular}{lcccccc}
\hline Feature & All (n=18) & $\begin{array}{c}\text { Non-cirrhotic } \\
(\mathrm{n}=8)\end{array}$ & $\begin{array}{c}\text { Cirrhotic } \\
(\mathrm{n}=10)\end{array}$ & All (n=24) & $\begin{array}{c}\text { Non-cirrhotic } \\
(\mathrm{n}=14)\end{array}$ & $\begin{array}{c}\text { Cirrhotic } \\
(\mathrm{n}=10)\end{array}$ \\
\hline Age (years) & $54.2 \pm 8.2$ & $55.6 \pm 6.4$ & $44.7 \pm 8.6$ & $60.1 \pm 11.1$ & $59.4 \pm 12.6$ & $61.2 \pm 9.2$ \\
Duration of PBC (years) & $5.9 \pm 5.2$ & $3.0 \pm 3.7$ & $8.3 \pm 5.1$ & $6.6 \pm 4.4$ & $4.8 \pm 3.5$ & $8.6 \pm 4.6$ \\
ALP (IU/l) & $214 \pm 80.1$ & $204 \pm 104.8$ & $221 \pm 58.8$ & $173 \pm 115.6$ & $154 \pm 98.0$ & $200 \pm 133.6$ \\
GGT (IU/l) & $173 \pm 171.5$ & $189 \pm 210.6$ & $160 \pm 143.7$ & $108 \pm 89.8$ & $109 \pm 96.8$ & $105 \pm 77.5$ \\
Bilirubin (mg/dl) & $1.5 \pm 1.3$ & $0.6 \pm 0.2$ & $2.3 \pm 1.2$ & $1.5 \pm 1.9$ & $0.5 \pm 0.1$ & $2.8 \pm 2.3$ \\
ALT (IU/l) & $62 \pm 49.4$ & $45 \pm 23.3$ & $76 \pm 60.8$ & $46 \pm 26.6$ & $35 \pm 14.6$ & $62 \pm 31.6$ \\
AST (IU/l) & $63 \pm 28.1$ & $44 \pm 12.4$ & $78 \pm 28.2$ & $54 \pm 46.6$ & $33 \pm 12.6$ & $82 \pm 61.1$ \\
Albumin (g/dl) & $4.1 \pm 0.4$ & $4.5 \pm 0.2$ & $3.8 \pm 0.3$ & N/A & N/A & N/A \\
INR & $1.1 \pm 0.2$ & $1.0 \pm 0.1$ & $1.2 \pm 0.1$ & N/A & N/A & N/A \\
Total cholesterol (mg/dl) & $227 \pm 65.7$ & $256 \pm 49.5$ & $203 \pm 69.7$ & N/A & N/A & N/A \\
\hline
\end{tabular}

PBC: primary biliary cholangitis; ALT: alanine aminotransferase; AST: aspartate aminotransferase; ALP: alkaline phosphatase; GGT: gamma-glutamyl transferase; INR: International Normalized Ratio; N/A: non applicable.

Data presented as the arithmetic means \pm SD. At the baseline demographic data and liver biochemistry were comparable in the study and in the control groups.

reduction of bilirubin level was noted. When all patients treated with SAMe were considered there was a significant reduction of total bilirubin and GGT levels. In the control group there were no changes in liver function tests. Data on liver biochemistry in all analysed groups are summarized in Table II. Withdrawal of SAMe caused almost uniform increase of liver biochemistry taken one month later but it did not reach statistical significance (Supplementary Fig. 1).

Effect of SAMe on sulfation of LCA and FGF-19 levels

Serum LCA and LCA-S concentrations were used to estimate the sulfation capacity of the liver (LCA/LCA-S ratio).
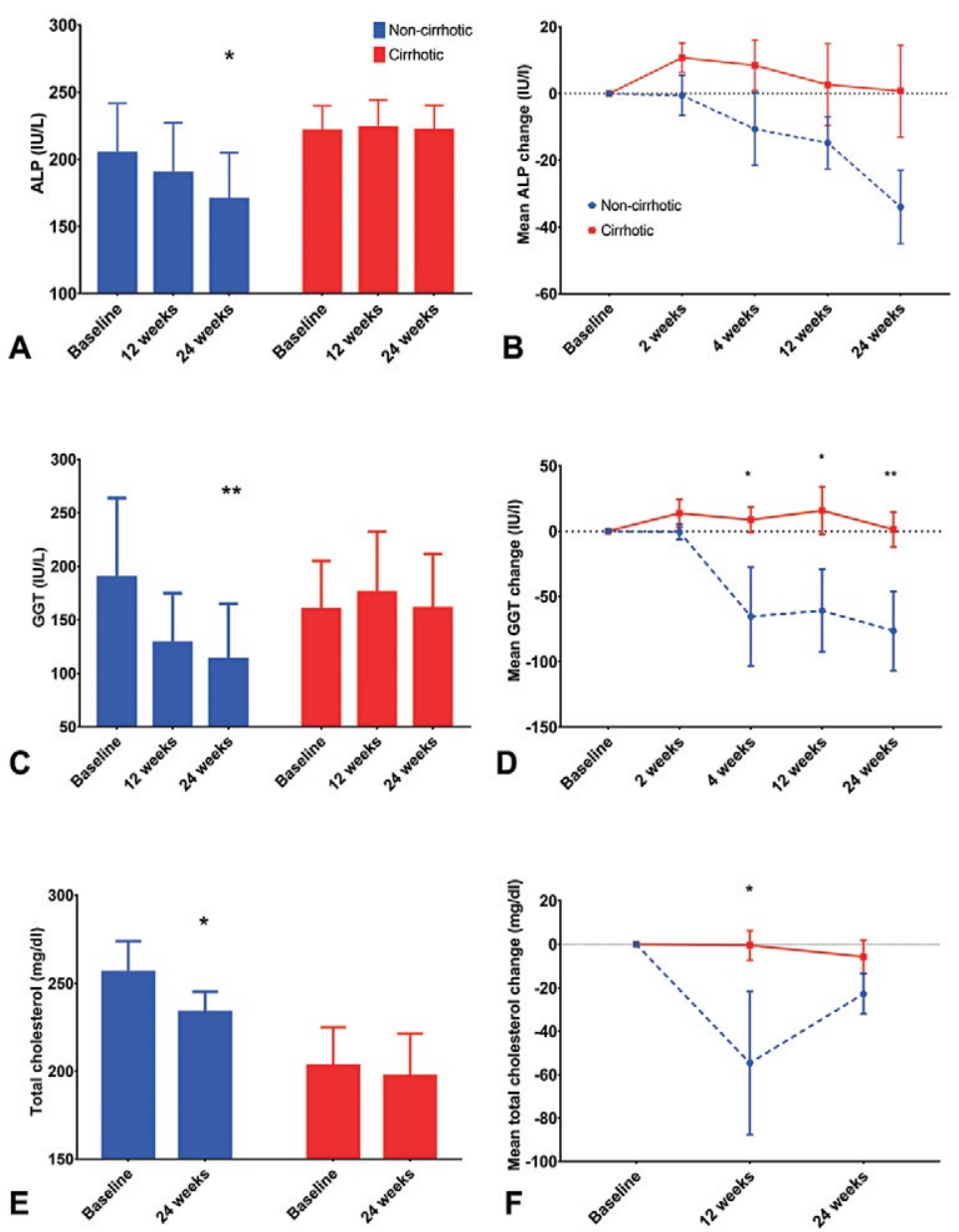

Fig. 1. Serum ALP (A), GGT (C) and total cholesterol (E) levels throughout the study period in non-cirrhotic and cirrhotic patients treated with SAMe. In non-cirrhotic patients serum ALP, GGT and total cholesterol levels decreased after 24 weeks of SAMe treatment. Absolute changes in serum ALP (B), GGT (D) and total cholesterol (F) levels throughout the study period in non-cirrhotic and cirrhotic patients treated with SAMe. In non-cirrhotic patients mean absolute changes in GGT and cholesterol levels were significantly higher than in cirrhotic patients. $\mathrm{Y}$ axis indicates absolute changes compared to baseline levels. Continuous variables are summarized using the arithmetic mean and the standard error of the mean (SEM). Two-sided $p$ values were calculated using paired t-tests or Wilcoxon rank sum tests in comparison to baseline. ${ }^{*}$ indicates $\mathrm{p}<0.05$ and ${ }^{*}$ indicates $\mathrm{p}<0.01$. 
Table II. Liver biochemistry at baseline and after 24 weeks in the analysed groups of patients

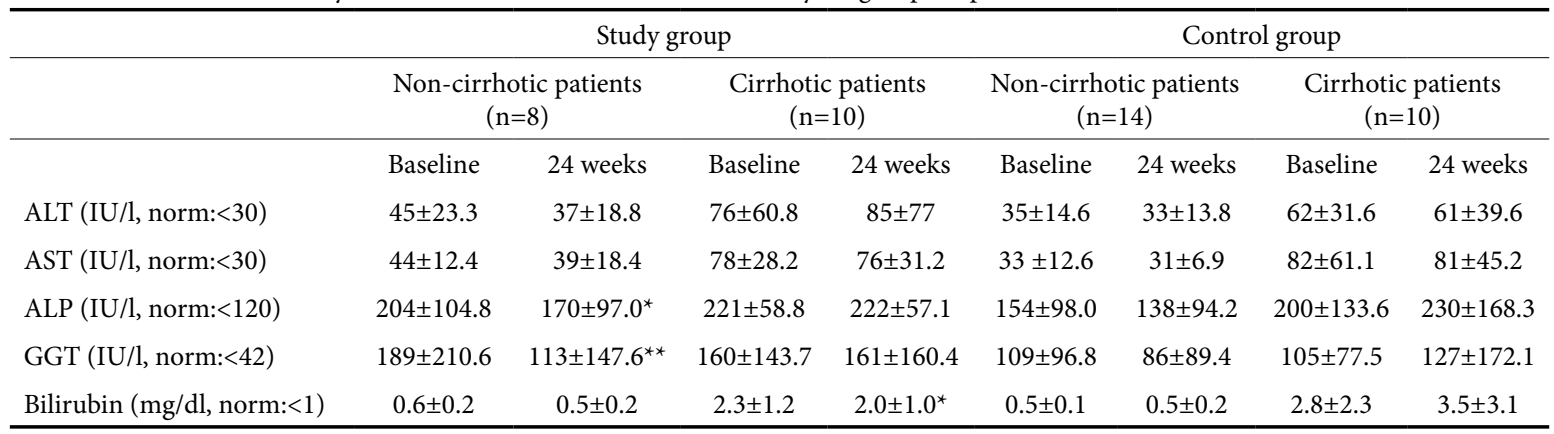

ALT: alanine aminotransferase; AST: aspartate aminotransferase; ALP: alkaline phosphatase; GGT: gamma-glutamyl transferase. Data presented as the arithmetic mean \pm SD. Two-sided $\mathrm{p}$ values were calculated using paired t-tests or Wilcoxon rank sum tests. $\mathrm{p}$ values in comparison to baseline: ${ }^{*} \mathrm{p}<0.05,{ }^{* *} \mathrm{p}<0.01,{ }^{* *} \mathrm{p}<0.001$

We observed an enhancement of sulfated derivative of LCA in patients treated with SAMe; however, these changes did not reach statistical significance (LCA/LCA-S ratio $4.8 \pm 8.1$ at baseline vs $2.8 \pm 3.4$ at 24 weeks; $\mathrm{p}=0.55$ ). In order to evaluate SAMe capacity to reduce cholestatic liver injury we measured serum FGF-19 levels. We did not observe any changes between baseline and end-point FGF-19 values in both non-cirrhotic $(162.9 \pm 160.1$ at baseline vs $162.2 \pm 152.9 \mathrm{pg} / \mathrm{ml}$ at 24 weeks; $\mathrm{p}=0.99)$ and cirrhotic patients treated with SAMe (176.0 \pm 181.9 at baseline vs $149.6 \pm 124.7 \mathrm{pg} / \mathrm{ml}$ at 24 weeks; $\mathrm{p}=0.3$ ).

\section{Effect of SAMe on the HRQoL}

In line with the enhancement of markers of liver injury the therapy with SAMe led to the improvement of the patients' well-being. In particular, a significant improvement of fatigue and pruritus measured by PBC-40 questionnaire as well as emotional distress in STAI 2 was seen when scorings from baseline and end of treatment were compared. There was also a trend towards the improvement of MFIS scores and Depression and Anxiety domains of HADS without reaching statistical significance. These data are summarized in Table III.

\section{DISCUSSION}

SAMe plays a crucial role in several biochemical processes exerting its protective effect in various cells. Several conditions that increase oxidative stress, hypoxia or nitric oxide synthesis, which include viral hepatitis, alcoholic liver disease or various toxins disturb synthesis of SAMe via a direct effect on methionine adenyltransferase, i.e. the enzyme responsible for SAMe synthesis from methionine [17]. We have recently shown that protection against oxidative stress mediated by Nrf2/Keap1 pathway is also significantly impaired in PBC [18].

SAMe has been extensively studied in a large number of animal and experimental studies. Its supplementation leads to increased intracellular concentration of glutathione both in experimental models [19] and in patients with liver diseases [20]. It prevented liver hypoxia and restored activity of mitochondrial respiratory complexes in a rat model of alcohol induced fatty liver [21].

In terms of using SAMe in humans, literature data are scanty. A recent meta-analysis screened almost 2000 articles (both animal and human studies) selecting only 11 studies as being suitable for the analysis [22]. Strikingly, in all but two
Table III. Results of HRQoL questionnaires in the study group

\begin{tabular}{|c|c|c|c|}
\hline & Baseline & 24 weeks & $\mathrm{p}$ value \\
\hline \multicolumn{4}{|c|}{ PBC- 40} \\
\hline Other symptoms & $18.6 \pm 4.0$ & $17.4 \pm 4.7$ & 0.15 \\
\hline Itch & $6.9 \pm 3.5$ & $5.2 \pm 2.3$ & 0.006 \\
\hline Fatigue & $30.2 \pm 7.8$ & $26.9 \pm 7.4$ & 0.04 \\
\hline Cognitive & $12.8 \pm 6.2$ & $12.1 \pm 6.5$ & 0.23 \\
\hline Social and emotional & $35.9 \pm 9.1$ & $32.7 \pm 7.7$ & 0.13 \\
\hline \multicolumn{4}{|c|}{ STAI } \\
\hline STAI 1 & $42.9 \pm 8.1$ & $42.2 \pm 7.2$ & 0.71 \\
\hline STAI 2 & $47.8 \pm 5.8$ & $44.6 \pm 6.0$ & 0.003 \\
\hline \multicolumn{4}{|c|}{ MFIS } \\
\hline Physical & $19.4 \pm 7.4$ & $16.6 \pm 7.2$ & 0.12 \\
\hline Cognitive & $13.7 \pm 8.4$ & $11.7 \pm 7.8$ & 0.25 \\
\hline Psychosocial & $3.5 \pm 1.4$ & $2.9 \pm 2.0$ & 0.22 \\
\hline Total Score & $35.9 \pm 15.3$ & $31.2 \pm 14.8$ & 0.15 \\
\hline \multicolumn{4}{|c|}{ HADS } \\
\hline HADS Depression & $5.3 \pm 2.6$ & $4.2 \pm 3.4$ & 0.13 \\
\hline HADS Anxiety & $7.8 \pm 3.8$ & $6.4 \pm 3.5$ & 0.12 \\
\hline
\end{tabular}

MFIS: Modified Fatigue Impact Scale; HADS: Hospital Anxiety and Depression Scale; STAI: State-Trait Anxiety Inventory. Data presented as the arithmetic mean $\pm \mathrm{SD}$. Two-sided $\mathrm{p}$ values were calculated using paired $t$-tests or Wilcoxon rank sum tests.

papers, SAMe was administered for a period of equal or less than 4 weeks, and a predominant indication was obstetric cholestasis. Considering the mode of action of SAMe it may seem unrealistic to expect its detectable effect within such a short time. Only in one study depicted by the above metaanalysis the treatment with SAMe was administered for a prolonged period of time. Mato et al. [4] in a multicentre, randomized, double blind study including a group of 123 patients with alcoholic cirrhosis applied SAMe in the dose of $1200 \mathrm{mg} /$ daily for the period of 24 months. They demonstrated that in patients with Child A and B cirrhosis, SAMe exerted a significant effect by reducing the death rates or the number of patients requiring liver transplantation. In a group treated with SAMe, only $12 \%$ of patients reached end points (i.e. overall mortality/liver transplantation) as compared to $29 \%$ in a placebo group $(\mathrm{p}=0.025)$. SAMe also significantly affected two-year survival ( $85 \%$ vs $67 \%$ in placebo group). Treatment 
was well tolerated and no severe adverse effects were reported. The second longest trial mentioned in the meta-analysis was a randomized, double blind study undertaken by Medici et al. [5]. It comprised 37 subjects with alcoholic liver disease, 18 of them received SAMe and 19 placebo. The SAMe dose of 1200 $\mathrm{mg} /$ daily was identical to the one used in the Mato et al. [4] study and it was administered over the period of 6 months. An improvement of liver biochemistry in both groups was seen, likely related to alcohol abstinence, and it was not significantly better in SAMe group than in the placebo one. This perhaps should not be a surprise when we compared these results with the study of Mato et al. [4] on patients with the same etiology, where no effect was seen after 6 months of SAMe treatment. Indeed, Medici et al. [5] study seems to confirm the notion that SAMe ideally should be used for a longer period of time, at least in patients with alcoholic etiology. This concept may somehow be supported by the results of the study by Feld et al. [23]. They clearly demonstrated that adding SAMe in the dose of $800 \mathrm{mg}$ bd to the treatment of hepatitis $\mathrm{C}$ with pegylated interferon and ribavirin over a period of 12 months increased interferon stimulated gene in non-responders to previous therapy. The in vitro work included in this study [23] showed that SAMe improved interferon signalling and its antiviral effect. This phenomenon was linked to enhanced metylation induced by SAMe. As there is only one published randomized study where SAMe was used as a single agent for a period longer than 6 months, one has to agree with the conclusion of the review paper which said that "to date no large, high quality, randomized placebo-controlled clinical trials have been performed that definitely establish clinical utility (of SAMe) in specific disease states" [24].

In this pilot, proof of the principle and open label work we studied the effect of SAMe on liver biochemistry and HRQoL in a small group of consecutive patients with PBC who were treated with UDCA for a period of at least 6 months. A rationale for this analysis was based on our laboratory studies performed almost two decades ago when we used a hepatocyte couplets model for the study of hepatoprotection in experimentally induced cholestasis $[6,7,25,26]$. We demonstrated both protective and reversing effect of SAMe on lithocholate and $17 \beta$-estradiol glucuronide induced cholestasis. This effect was additive in the former when UDCA and SAMe were used simultaneously. As UDCA and SAMe exert their hepatoprotective effects by different molecular and biochemical mechanisms, it is conceivable that when used together, their effect may potentially be additive. We have shown that the hepatoprotective effect of SAMe, at least partially and in experimental models, may be related to its role as a sulphate donor, as the anticholestatic effect of SAMe was ameliorated by dehydroepiandrosterone, which competes for sulphate group with $17 \beta$-estradiol glucuronide. Sulphation of the latter makes it more water soluble and non-cholestatic.

These present results did not, however, confirm our hypothesis on a potential role of SAMe as a sulphate donor. Although we observed an enhancement of sulphated derivative of LCA in both cirrhotic and non-cirrhotic patients treated with SAMe, these changes did not reach statistical significance. Treatment with SAMe had also no effect on serum levels of FGF-19, which as we demonstrated before, correlate with
Mayo Risk Score and are significantly increased in UDCA non-responders [12]. These findings may be related to the small size of the analysed cohort and, in our opinion, require further studies on a larger group of patients.

In the present study we observed a significant improvement in ALP, GGT and total cholesterol in non-cirrhotic patients. This may suggest that SAMe exerts its effect particularly in earlier stages of the disease and it may somehow correspond with the results of the Mato et al. [4] study where SAMe showed no effect in patients with advanced disease and the Child C score.

HRQoL has emerged as one of the most important issues in contemporary medicine. Improvement in liver biochemistry excites treating physicians, but from the patients' perspective does not mean much if it is not associated with improved QoL. Some treatments may clearly improve the liver tests but simultaneously have no effect or even affect negatively patients' wellbeing, a good example being steroids in autoimmune hepatitis. Chronic fatigue and pruritus are considered to be the most debilitating symptoms in PBC. Treatment with SAMe caused a significant improvement of both symptoms. Certainly, these results have to be interpreted with caution. First of all, patients included into the study did not have a significant itching and did not use antipruritic agents over the period of study. Two patients with more pronounced itching were withdrawn within one month as they did not observe any improvement of their pruritus and were put on antipruritic treatment. Thus, a statistically significant improvement of pruritus is a surprise, but on the other hand a positive effect of SAMe on itching was reported in a randomized study in obstetric cholestasis [27]. We have also seen a significant improvement caused by SAMe on the fatigue domain of PBC-40 questionnaire. SAMe has a well described antidepressive properties, which are comparable to selective serotonin reuptake inhibitors (SSRIs) [28]. Although SSRIs did not prove effective in the treatment of fatigue in PBC in a small randomized study [29], antidepressants are frequently prescribed in fatigued patients with PBC [30]. A recent review strongly suggested that SAMe might prove useful in various psychiatric conditions including not only depression but also mood disturbance or anxiety [31]. In our patients treated with SAMe we observed a significant improvement in the STAI 2 questionnaire, which is a well validated tool for the assessment of anxiety. We have also seen a trend towards improvement in the Modified Fatigue Impact Scale and Depression and Anxiety domain of HADS but certainly it is too early to draw any firm conclusions from these findings. Four patients were removed from the study because of unspecific symptoms, mostly abdominal cramps. As SAMe in the dose we used or $800 \mathrm{mg}$ bd was well tolerated in other studies, this finding was indeed unexpected. The severity of symptoms was mild/moderate and perhaps not related to the treatment (one third of the patients with $\mathrm{PBC}$ report unspecific right upper abdominal quadrant pain); however, we decided to remove these patients from the study after 4 weeks.

\section{CONCLUSIONS}

In this pilot study on a small cohort of patients with $\mathrm{PBC}$ we observed a significant improvement of liver biochemistry 
caused by SAMe in non-cirrhotics and a clear positive effect on HRQoL. In our opinion, these early results warrant further study on a larger cohort of patients to characterize a potential positive effect of SAMe in patients with chronic cholestatic liver conditions.

Conflicts of interest: The authors declare no conflict of interest and state there is no pertinent financial arrangement.

Authors' contributions: E.W.: study design, acquisition, analysis and interpretation of data, drafting of the manuscript, statistical analysis. administrative support. J.R.W.: acquisition, analysis and interpretation of data. M.K.: analysis and interpretation of data, critical revision of the manuscript for important intellectual content, statistical analysis. O.B.: acquisition analysis and interpretation of data, critical revision of the manuscript for important intellectual content, technical and material support. M.M.: acquisition, analysis and interpretation of data, critical revision of the manuscript for important intellectual content, statistical analysis. P.M.: study concept and design, analysis and interpretation of data, drafting of the manuscript, obtaining funding, study supervision.

Acknowledgement: This work was supported by grant no. 2011/02/A/ NZ5/00321 from the National Science Centre in Poland.

Supplementary material: To access the supplementary material visit the online version of the J Gastrointestin Liver Dis at http://www. jgld.ro/wp/archive/y2018/n3/a12 and http://dx.doi.org/10.15403/ jgld.2014.1121.273.icz

\section{REFERENCES}

1. Milkiewicz P, Heathcote EJ. Fatigue in chronic cholestasis. Gut 2004;53:475-477. doi:10.1136/gut.2003.025155

2. European Association for the Study of the Liver. EASL Clinical Practice Guidelines: The diagnosis and management of patients with primary biliary cholangitis. J Hepatol 2017;67:145-172. doi:10.1016/j. jhep.2017.03.022

3. Lu SC, Mato JM. S-adenosylmethionine in liver health, injury, and cancer. Physiol Rev 2012;92:1515-1542. doi:10.1152/physrev.00047.2011

4. Mato JM, Camara J, Fernandez de Paz J, et al. S-adenosylmethionine in alcoholic liver cirrhosis: a randomized, placebo-controlled, double-blind, multicenter clinical trial. J Hepatol 1999;30:1081-1089. doi:10.1016/S0168-8278(99)80263-3

5. Medici V, Virata MC, Peerson JM, et al. S-adenosyl-L-methionine treatment for alcoholic liver disease: a double-blinded, randomized placebo-controlled trial. Alcohol Clin Exp Res 2011;35:1960-1965. doi:10.1111/j.1530-0277.2011.01547.x

6. Milkiewicz P, Mills CO, Roma MG, Ahmed-Choudhury J, Elias E, Coleman R. Tauroursodeoxycholate and S-adenosyl-L-methionine exert an additive ameliorating effect on taurolithocholate-induced cholestasis: a study in isolated rat hepatocyte couplets. Hepatology 1999;29:471-476. doi:10.1002/hep.510290215

7. Milkiewicz P, Roma MG, Cardenas R, Mills CO, Elias E, Coleman R. Effect of tauroursodeoxycholate and S-adenosyl-L-methionine on 17beta-estradiol glucuronide-induced cholestasis. J Hepatol 2001;34:184-191. doi:10.1016/S0168-8278(00)00066-0
8. Milkiewicz P, Roma MG, Elias E, Coleman R. Pathobiology and experimental therapeutics in hepatocellular cholestasis: lessons from the hepatocyte couplet model. Clin Sci (Lond) 2002;102:603-614. doi:10.1042/cs1020603

9. Herrmann E, de Ledinghen V, Cassinotto C, et al. Assessment of biopsyproven liver fibrosis by 2D-shear wave elastography: An individual patient data based meta-analysis. Hepatology 2018;67:260-272. doi:10.1002/hep.29179

10. Trottier J, Bialek A, Caron P, Straka RJ, Milkiewicz P, Barbier O. Profiling circulating and urinary bile acids in patients with biliary obstruction before and after biliary stenting. PLoS One 2011;6:e22094. doi:10.1371/ journal.pone.0022094

11. Trottier J, Bialek A, Caron P, et al. Metabolomic profiling of 17 bile acids in serum from patients with primary biliary cirrhosis and primary sclerosing cholangitis: a pilot study. Dig Liver Dis 2012;44:303-310. doi:10.1016/j.dld.2011.10.025

12. Wunsch E, Milkiewicz M, Wasik U, et al. Expression of hepatic Fibroblast Growth Factor 19 is enhanced in Primary Biliary Cirrhosis and correlates with severity of the disease. Sci Rep 2015;5:13462. doi: $10.1038 /$ srep 13462

13. Jacoby A, Rannard A, Buck D, et al. Development, validation, and evaluation of the PBC-40, a disease specific health related quality of life measure for primary biliary cirrhosis. Gut 2005;54:1622-1629. doi:10.1136/gut.2005.065862

14. Raszeja-Wyszomirska J, Wunsch E, Krawczyk M, et al. Assessment of health related quality of life in polish patients with primary biliary cirrhosis. Clin Res Hepatol Gastroenterol 2016;40:471-479. doi:10.1016/j.clinre.2015.10.006

15. Fisk JD, Ritvo PG, Ross L, Haase DA, Marrie TJ, Schlech WF. Measuring the functional impact of fatigue: initial validation of the fatigue impact scale. Clin Infect Dis 1994;18 Suppl 1:S79-S83. doi:10.1093/clinids/18. Supplement_1.S79

16. Zigmond AS, Snaith RP. The hospital anxiety and depression scale. Acta Psychiatr Scand 1983;67:361-370. doi:10.1111/j.1600-0447.1983. tb09716.x

17. Mato JM, Corrales FJ, Lu SC, Avila MA. S-Adenosylmethionine: a control switch that regulates liver function. FASEB J 2002;16:15-26. doi:10.1096/fj.01-0401rev

18. Wasik U, Milkiewicz M, Kempinska-Podhorodecka A, Milkiewicz P. Protection against oxidative stress mediated by the Nrf2/Keap1 axis is impaired in Primary Biliary Cholangitis. Sci Rep 2017;7:44769. doi: $10.1038 / \operatorname{srep} 44769$

19. Garcia-Ruiz C, Morales A, Colell A, et al. Feeding S-adenosyl-Lmethionine attenuates both ethanol-induced depletion of mitochondrial glutathione and mitochondrial dysfunction in periportal and perivenous rat hepatocytes. Hepatology 1995;21:207-214. doi:10.1002/ hep.1840210133

20. Vendemiale G, Altomare E, Trizio T, et al. Effects of oral S-adenosyl-Lmethionine on hepatic glutathione in patients with liver disease. Scand J Gastroenterol 1989;24:407-415. doi:10.3109/00365528909093067

21. King AL, Mantena SK, Andringa KK, et al. The methyl donor S-adenosylmethionine prevents liver hypoxia and dysregulation of mitochondrial bioenergetic function in a rat model of alcoholinduced fatty liver disease. Redox Biol 2016;9:188-197. doi:10.1016/j. redox.2016.08.005

22. Guo T, Chang L, Xiao Y, Liu Q. S-adenosyl-L-methionine for the treatment of chronic liver disease: a systematic review and meta-analysis PLoS One 2015;10:e0122124. doi:10.1371/journal.pone.0122124 
23. Feld JJ, Modi AA, El-Diwany R, et al. S-adenosyl methionine improves early viral responses and interferon-stimulated gene induction in hepatitis C nonresponders. Gastroenterology 2011;140:830-839. doi:10.1053/j.gastro.2010.09.010

24. Anstee QM, Day CP. S-adenosylmethionine (SAMe) therapy in liver disease: a review of current evidence and clinical utility. J Hepatol 2012;57:1097-1109. doi:10.1016/j.jhep.2012.04.041

25. Milkiewicz P, Roma MG, Elias E, Coleman R. Hepatoprotection with tauroursodeoxycholate and beta muricholate against taurolithocholate induced cholestasis: involvement of signal transduction pathways. Gut 2002;51:113-119. doi:10.1136/gut.51.1.113

26. Roma MG, Milkiewicz P, Elias E, Coleman R. Control by signaling modulators of the sorting of canalicular transporters in rat hepatocyte couplets: role of the cytoskeleton. Hepatology 2000;32:1342-1356. doi:10.1053/jhep.2000.20519

27. Roncaglia N, Locatelli A, Arreghini A, et al. A randomised controlled trial of ursodeoxycholic acid and S-adenosyl-1-methionine in the treatment of gestational cholestasis. BJOG 2004;111:17-21. doi:10.1046/ j.1471-0528.2003.00029.x

28. Sarris J, Papakostas GI, Vitolo O, Fava M, Mischoulon D. S-adenosyl methionine (SAMe) versus escitalopram and placebo in major depression RCT: efficacy and effects of histamine and carnitine as moderators of response. J Affect Disord 2014;164:76-81. doi:10.1016/j. jad.2014.03.041

29. ter Borg PC, van Os E, van den Broek WW, Hansen BE, van Buuren HR. Fluvoxamine for fatigue in primary biliary cirrhosis and primary sclerosing cholangitis: a randomised controlled trial [ISRCTN88246634]. BMC Gastroenterol 2004;4:13. doi:10.1186/1471-230X-4-13

30. Al-Harthy N, Kumagi T, Coltescu C, Hirschfield GM. The specificity of fatigue in primary biliary cirrhosis: evaluation of a large clinic practice. Hepatology 2010;52:562-570. doi:10.1002/hep.23683

31. Sharma A, Gerbarg P, Bottiglieri T, et al. S-Adenosylmethionine (SAMe) for Neuropsychiatric Disorders: A Clinician-Oriented Review of Research. J Clin Psychiatry 2017;78:e656-e667. doi:10.4088/JCP.16r11113 

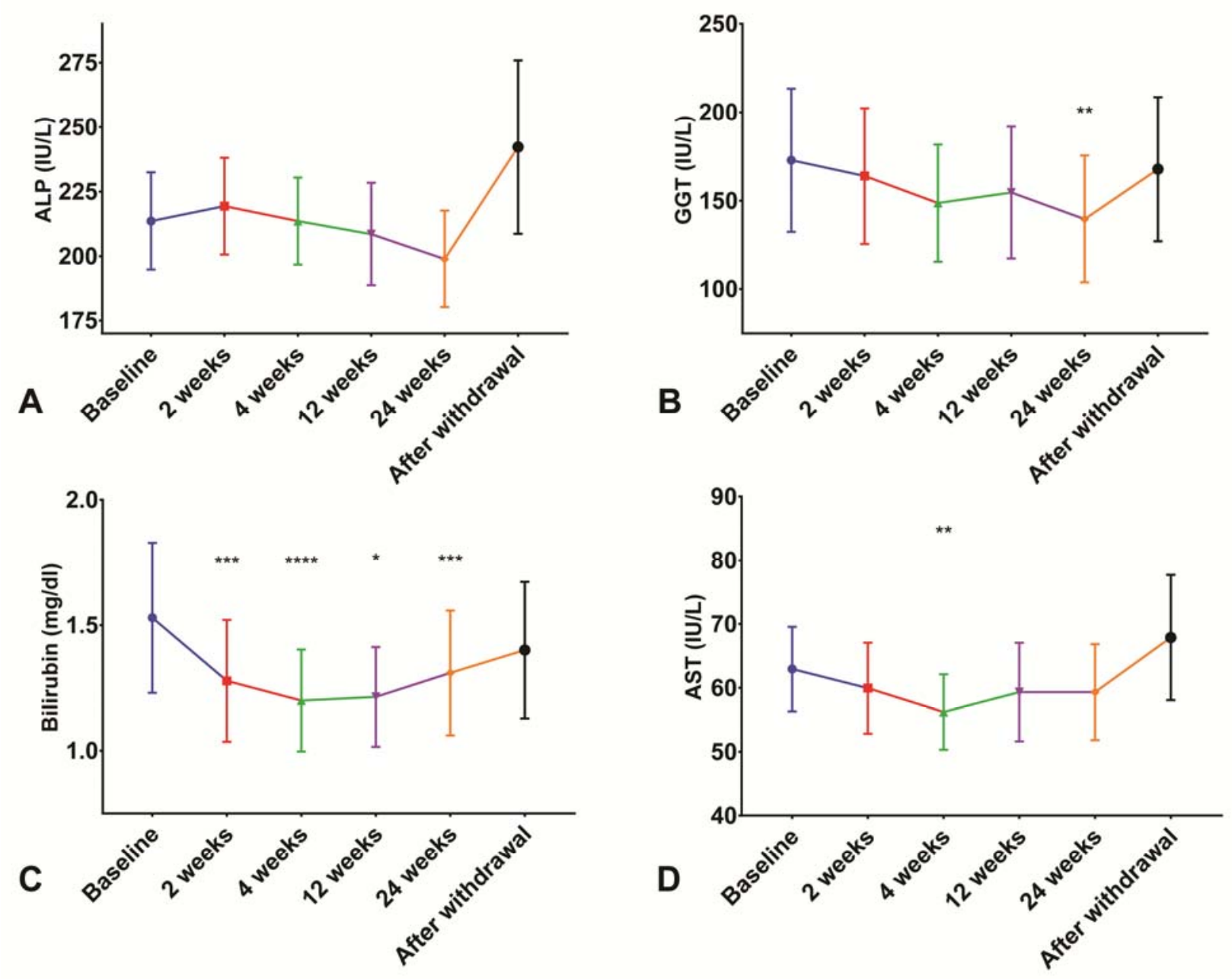

Supplementary Fig. 1. Absolute changes in serum ALP (A), GGT (B), total bilirubin (C) and AST (D) levels throughout the study period in all patients treated with SAMe. During the treatment period a significant improvement of GGT, total bilirubin and AST levels was noted. Withdrawal of SAMe caused a uniform increase of liver biochemistry but it did not reach statistical significance. Y axis indicates absolute changes compared to baseline levels. Continuous variables are summarized using the arithmetic mean and the standard error of the mean (SEM). Two-sided $p$ values were calculated using paired t-tests or Wilcoxon rank sum tests in comparison to baseline. * indicates $p<0.05$, ** indicates $p<0.01^{* * *}$, indicates $p<0.001$ and $* * * *$ indicates $p<0.0001$. 